\title{
Genetic risk factors influenced cocaine abuse and dependence more than cocaine use in women
}

\author{
Kendler KS, Prescott CA. Cocaine use, abuse and dependence in a population-based sample of female twins. BrJ Psychiatry 1998 \\ Oct;173:345-50.
}

\section{Question}

How much influence do genetic and environmental risk factors have on lifetime cocaine use, abuse, and dependence in women?

\section{Design}

A population based, cohort study using data from a twin registry.

\section{Setting}

Virginia, USA.

\section{Participants}

1937 of 2288 eligible white women (mean age 37 y) were interviewed. Participants were members of female-female pairs listed in the Virginia Twin Registry. Data for 1934 women were included in the analysis; both twins were included for 485 monozygotic (MZ) pairs and 335 (DZ) dizygotic pairs.

\section{Assessment of risk factors \\ Zygosity was determined blindly by standard questions, photo- graphs, and DNA, when necessary. Telephone interviewers, who were blind to information about the co-twin, asked questions about childhood (how often the twins shared the same room at home, were in the same class at school, and were dressed alike), adolescence (how often they had the same friends, were in the same social group, and went out together to films and dances), and adulthood (how much contact they had had in the previous year). These data were used to assess the similarity of environments for each pair of twins.}

\section{Main outcome measures}

Lifetime cocaine use, abuse, and dependence were assessed by an adapted Structured Clinical Interview for DSM-III-R, patient version interview. Cocaine abuse and dependence were diagnosed by using $D S M-I V$ criteria.

\begin{abstract}
Main results
$\mathrm{MZ}$ and DZ twins whose co-twins had used cocaine were more likely to use cocaine than those whose co-twins had not used cocaine (odds ratio [OR] 14.2, 95\% CI 8.7 to 23.2 for MZ twins and OR 6.7, CI 3.9 to 11.7 for DZ twins). MZ twins were also more likely to abuse or be dependent on cocaine if their co-twins had abused or been dependent on cocaine (OR 40.8, CI 16.3 to 102.4 for cocaine abuse and OR 27.6, CI 9.4 to 81.5 for cocaine dependence). For DZ twins, cocaine abuse and dependence did not have statistically significant associations with cocaine abuse (OR 2.70, CI 0.6 to 13.1) or dependence in the co-twin (OR not estimated because no DZ twins were concordant). Tetrachoric correlations were high for cocaine use, abuse, and dependence in MZ twins $(r=0.73, r=0.80$, and $r=0.68$, respectively). In DZ twins, the correlation was greater for cocaine use $(r=0.54)$ than for cocaine abuse $(r=0.18)$ or dependence $(r=0.08)$. Models that were developed to fit the data showed that cocaine abuse and dependence were influenced mainly by genetic risk factors, which explained $79 \%$ of the variance for abuse and $65 \%$ of the variance for dependence. For cocaine use, 39\% of the variance was explained by genetic and $35 \%$ by environmental risk factors.
\end{abstract}

\section{Conclusions}

Lifetime cocaine abuse and dependence in women were mainly influenced by genetic risk factors. Lifetime cocaine use in women was influenced partly by genetic and partly by environmental risk factors.

Source of funding: National Institutes of Health. For correspondence: Dr K S Kendler, Department of Psychiatry, Medical College of Virginia, Virginia
Commonwealth University, Box 980126, Richmond, VA 23298-0126, USA. Fax +18048281472.

\section{Commentary}

Family, twin, and adoption studies are the cornerstone of genetic epidemiology. In psychiatry, these paradigms enhance our understanding of putative causal mechanisms, although direct evidence for genetic and environmental determinants remains to be elucidated for the major psychiatric disorders. Many in the field are optimistic about the recent advances in molecular biology and believe that the identification of genetic factors is within our reach for some of these disorders. Evidence from family, twin, and adoption studies are essential stepping stones for the identification of genetic factors to be investigated further in linkage and segregation studies.

A combination of these approaches has led to compelling evidence for the role of genetic factors in substance abuse (as reviewed in the study by Kendler $e t a l$ ). This study provides an additional piece of this complex puzzle and is a major contri- bution to the literature because it is the first reported twin study of cocaine use and abuse in women.

The strengths of this study lie in its excellent methodology. Study strengths include (a) the community based twin sample, which enhances the generalisability of the findings, (b) the blinding of interviewers, (c) the use of standardised diagnostic criteria, (d) the examination of multiple sources of potential bias that might attenuate or diminish effect sizes, and (e) the statistical approach used to show twin resemblance for liability to cocaine use and abuse.

The inherent weakness of this study, as reported by the authors, is the small number of twins with abuse and dependence. Although the proportions of cocaine use, abuse, and dependence are similar to what is reported in the National Comorbidity Survey, the small number of affected twins makes it difficult to estimate the magnitude of the genetic effect. It is clear that these findings need to be replicated in a larger sample of female twins. It would be of additional clinical interest to conduct a longitudinal study to determine whether the course and outcome differs in men and women.

The clinical implications of these findings for practice are subtle, yet important. They include (a) the need for establishing family history of substance use and abuse, and (b) the need to gather information during clinical interviews to distinguish between use and abuse of substances. These 2 sources of information may be particularly important if future studies show that the magnitude of the genetic effect is associated with differential response to treatment

Denise Stevens, MSc, $\mathrm{PhD}$ Yale University School of Medicine New Haven, Connecticut, USA 